\title{
On intuitionistic fuzzy prime submodules
}

\author{
P. K. Sharma ${ }^{1}$ and Gagandeep Kaur ${ }^{2}$ \\ ${ }^{1}$ Post Graduate Department of Mathematics, D.A.V. College \\ Jalandhar, Punjab, India \\ e-mail: pksharma@davjalandhar.com \\ ${ }^{2}$ Research Scholar, IKG PT University \\ Jalandhar, Punjab, India \\ e-mail: taktogagan@gmail.com
}

Received: 3 May $2018 \quad$ Revised: 7 November $2018 \quad$ Accepted: 11 November 2018

\begin{abstract}
In this paper, we study intuitionistic fuzzy prime submodules with the help of residual quotient subsets of ring and modules. Also a complete characterisation of an intuitionistic fuzzy prime submodule is given. A relationship between intuitionistic fuzzy prime submodule and intuitionistic fuzzy maximal submodule is established. Homeomorphic image and pre-image of intuitionistic fuzzy prime submodules are obtained.
\end{abstract}

Keywords: Intuitionistic fuzzy prime (maximal) submodule, Intuitionistic fuzzy prime (maximal) ideal, Residual quotients of intuitionistic fuzzy submodules.

2010 Mathematics Subject Classification: 03F55, 16D10, 46J20.

\section{Introduction}

The concept of intuitionistic fuzzy sets was introduced by Atanassov [3-5] as a generalization to the notion of fuzzy sets given by Zadeh [21]. Biswas was the first to introduce the intuitionistic fuzzification of the algebraic structures and developed the concept of intuitionistic fuzzy subgroup of a group in [8]. Hur and others in [11] defined and studied intuitionistic fuzzy subrings and ideals of a ring. In [10], Davvaz et al. introduced the notion of intuitionistic fuzzy submodules which was further studied by many mathematicians (see [7, 12,13,15-17, 19]).

The correspondence between certain ideals and submodules arising from residual quotient plays a vital role in the decomposition theory and Goldie like structures (see [9]). A detailed 
study of the fuzzification of this and related concepts can be found in [1,14]. Intuitionistic fuzzification of such crisp sets leads us to structures that can be termed as intuitionistic fuzzy prime submodules. In this paper, we attempt to define intuitionistic fuzzy prime ideal of a ring and intuitionistic fuzzy prime submodule of a module using the concept of residual quotients and investigate various characteristic of it. This concept will help us to explore and investigate various facts about the intuitionistic fuzzy aspects of associated primes, Godlie like structures and singular ideals.

\section{Preliminaries}

Throughout the paper, $R$ is a commutative ring with unity $1,1 \neq 0, M$ is a unitary $R$-module and $\theta$ is the zero element of $M$. A proper submodule $N$ of $M$ is called a prime submodule if $r m \in N$ for some $r \in R, m \in M$ implies that $m \in N$ or $r \in(N: M)$, where

$$
(N: M)=\{r \mid r \in R, r M \subseteq N\} .
$$

The set of all prime submodules of $M$ is denoted by $\operatorname{Spec}(M)$.

Lemma 2.1. ([2]) Let $N$ be a prime submodule of $R$-module $M$. Then

(i) $N$ is a prime submodule of $M$ if and only if $P=(N: M)$ is a prime ideal of $R$ and $R / P$ module $M / N$ is torsion free.

(ii) $(N: M)$ is a maximal ideal of $R$, then, $N$ is a prime submodule of $M$.

(iii) If $N$ is a maximal submodule of $M$, then, $N$ is a prime submodule of $M$.

Definition 2.2. ([4,15]) Let $X$ be a non-empty fixed set. An intuitionistic fuzzy set (IFS) $A$ in $X$ is an object having the form $A=\left\{\left\langle x, \mu_{A}(x), \nu_{A}(x)\right\rangle \mid x \in X\right\}$, where the functions $\mu_{A}$ : $X \rightarrow[0,1]$ and $\nu_{A}: X \rightarrow[0,1]$ denote the degree of membership (namely $\mu_{A}(x)$ ) and the degree of non-membership (namely $\nu_{A}(x)$ ) of each element $x \in X$ to the set $A$, respectively, and $0 \leq \mu_{A}(x)+\nu_{A}(x) \leq 1$ for each $x \in X$.

Remark 2.3. (i) When $\mu_{A}(x)+\nu_{A}(x)=1$, i.e., when $\nu_{A}(x)=1-\mu_{A}(x)=\mu_{A^{c}}(x)$, then, $A$ is called a fuzzy set.

(ii) We denote the IFS $A=\left\{\left\langle x, \mu_{A}(x), \nu_{A}(x)\right\rangle \mid x \in X\right\}$ by $A=\left(\mu_{A}, \nu_{A}\right)$.

(iii) The class of intuitionistic fuzzy subsets of $X$ is denoted by $\operatorname{IF} S(X)$.

For $A, B \in I F S(X)$ we say that $A \subseteq B$ if and only if $\mu_{A}(x) \leq \mu_{B}(x)$ and $\nu_{A}(x) \geq \nu_{B}(x)$ for all $x \in X$. Also, $A \subset B$ if and only if $A \subseteq B$ and $A \neq B$.

By an intuitionistic fuzzy point $(I F P) x_{(p, q)}$ of $X, x \in X$ and $p, q \in(0,1]$ such that $p+q \leq 1$, we mean $x_{(p, q)} \in \operatorname{IFS}(X)$ defined by

$$
x_{(p, q)}(y)= \begin{cases}(p, q), & \text { if } y=x \\ (0,1), & \text { if otherwise }\end{cases}
$$


If $x_{(p, q)}$ is an intuitionistic fuzzy point of $X$ and $x_{(p, q)} \subseteq A \in \operatorname{IFS}(X)$, we write $x_{(p, q)} \in A$. Let $A=\left(\mu_{A}, \nu_{A}\right)$ be an IFS of $X$ and $Y \subseteq X$. Then, the restriction of $A$ to the set $Y$ is an IFS $A_{Y}=\left(\mu_{A_{Y}}, \nu_{A_{Y}}\right)$ of $X$ and is defined as:

$$
\mu_{A_{Y}}(y)=\left\{\begin{array}{ll}
\mu_{A}(y), & \text { if } y \in Y \\
0, & \text { if otherwise }
\end{array} ; \quad \nu_{A_{Y}}(y)= \begin{cases}\nu_{A}(y), & \text { if } y \in Y \\
1, & \text { otherwise }\end{cases}\right.
$$

The intuitionistic fuzzy characterisitic function of $X$ with respect to a subset $Y$ is denoted by $\chi_{Y}$ and is defined as:

$$
\mu_{\chi_{Y}}(y)=\left\{\begin{array}{ll}
1, & \text { if } y \in Y \\
0, & \text { if otherwise }
\end{array} ; \quad \nu_{\chi_{Y}}(y)= \begin{cases}0, & \text { if } y \in Y \\
1, & \text { otherwise }\end{cases}\right.
$$

The following are two very basic definitions given in [11] and [18].

Definition 2.4. Let $A \in I F S(R)$. Then, $A$ is called intuitionistic fuzzy ideal $(I F I)$ of $R$ if for all $x, y \in R$, the following statements are satisfied:

(i) $\mu_{A}(x-y) \geq \mu_{A}(x) \wedge \mu_{A}(y)$;

(ii) $\mu_{A}(x y) \geq \mu_{A}(x) \vee \mu_{A}(y)$;

(iii) $\nu_{A}(x-y) \leq \nu_{A}(x) \vee \nu_{A}(y)$;

(iv) $\nu_{A}(x y) \leq \nu_{A}(x) \wedge \nu_{A}(y)$.

Definition 2.5. Let $A \in I F S(M)$. Then, $A$ is called intuitionistic fuzzy module $(I F M)$ of $M$ if for all $x, y \in M, r \in R$, the following statements are satisfied:

(i) $\mu_{A}(x-y) \geq \mu_{A}(x) \wedge \mu_{A}(y)$;

(ii) $\mu_{A}(r x) \geq \mu_{A}(x)$;

(iii) $\mu_{A}(\theta)=1$;

(iv) $\nu_{A}(x-y) \leq \nu_{A}(x) \vee \nu_{A}(y)$;

(v) $\nu_{A}(r x) \leq \nu_{A}(x)$;

(vi) $\nu_{A}(\theta)=0$.

Let $\operatorname{IFM} M(M)$ denote the set of all intuitionistic fuzzy $R$-modules of $M$ and $I F I(R)$ denote the set of all intuitionistic fuzzy ideals of $R$. We note that when $R=M$, then, $A \in I F M(M)$ if and only if $\mu_{A}(\theta)=1, \nu_{A}(\theta)=0$ and $A \in I F I(R)$.

Let $A \in I F S(M)$ and $p, q \in[0,1]$ with $p+q \leq 1$. Then, the set $A_{(p, q)}=\left\{x \in M \mid \mu_{A}(x) \geq\right.$ $p$ and $\left.\nu_{A}(x) \leq q\right\}$ is called the $(p, q)$-cut subset of $M$ with respect to $A$. In particular, we denote $A_{\left(\mu_{A}(\theta), \nu_{A}(\theta)\right)}$ by $A_{*}$. Of course, $A_{*}=\left\{x \in M \mid \mu_{A}(x)=\mu_{A}(\theta)\right.$ and $\left.\nu_{A}(x)=\nu_{A}(\theta)\right\}$. The support of an IFS A is denoted by $A^{*}$ and is defined as $A^{*}=\left\{x \in M \mid \mu_{A}(x)>0\right.$ and $\left.\nu_{A}(x)<1\right\}$. 
Definition 2.6. Let $C \in I F I(R)$ and $B \in I F M(M)$. Define the composition $C \circ B$, and product $C \cdot B$, respectively, as follows: For all $w \in M$,

$$
\mu_{C \circ B}(w)= \begin{cases}\sup \left[\mu_{C}(r) \wedge \mu_{B}(x)\right] & \text { if } w=r x, r \in R, x \in M \\ 0, & \text { if } w \text { is not expressible as } w=r x\end{cases}
$$

and

$$
\begin{gathered}
\nu_{C \circ B}(w)= \begin{cases}\inf \left[\nu_{C}(r) \vee \nu_{B}(x)\right] & \text { if } w=r x, r \in R, x \in M \\
1, & \text { if } w \text { is not expressible as } w=r x .\end{cases} \\
\mu_{C \cdot B}(w)= \begin{cases}\sup \left[\inf _{i=1}^{n}\left\{\mu_{C}\left(r_{i}\right) \wedge \mu_{B}\left(x_{i}\right)\right\}\right] & \text { if } w=\sum_{i=1}^{n} r_{i} x_{i}, r_{i} \in R, x_{i} \in M, n \in N \\
0, & \text { if } w \text { is not expressible as } w=\sum_{i=1}^{n} r_{i} x_{i}\end{cases}
\end{gathered}
$$

and

$$
\nu_{C \cdot B}(w)= \begin{cases}\inf \left[\sup _{i=1}^{n}\left\{\nu_{C}\left(r_{i}\right) \vee \nu_{B}\left(x_{i}\right)\right\}\right] & \text { if } w=\sum_{i=1}^{n} r_{i} x_{i}, r_{i} \in R, x_{i} \in M, n \in N \\ 1, & \text { if } w \text { is not expressible as } w=\sum_{i=1}^{n} r_{i} x_{i} .\end{cases}
$$

where as usual supremum and infimum of an empty set are taken to be 0 and 1 , respectively. Clearly, $C \circ B \subseteq C \cdot B$.

The following results in the lemma can be easily derived. It gives the basic operations between intuitionistic fuzzy ideals and intuitionistic fuzzy modules.

Lemma 2.7. Let $C \in \operatorname{IFI}(R), A, B \in I F M(M)$ Then:

(i) $C \cdot B \subseteq A$ if and only if $C \circ B \subseteq A$.

(ii) Let $r_{(s, t)} \in \operatorname{IFS}(R), x_{(p, q)} \in \operatorname{IFS}(M)$ be IFPs. Then, $r_{(s, t)} \circ x_{(p, q)}=(r x)_{(s \wedge p, t \vee q)}$.

(iii) If $\mu_{C}(0)=1, \nu_{C}(0)=0$, then, $C \cdot A \in \operatorname{IF} M(M)$.

(iv) Let $r_{(s, t)} \in \operatorname{IFS}(R)$ be an IFP. Then, for all $w \in M$,

$$
\mu_{r_{(s, t)}^{\circ} \circ}(w)= \begin{cases}\sup \left[s \wedge \mu_{B}(x)\right] & \text { if } w=r x, r \in R, x \in M \\ 0, & \text { if } w \text { is not expressible as } w=r x\end{cases}
$$

and

$$
\nu_{r_{(s, t)} \circ B}(w)= \begin{cases}\inf \left[t \vee \nu_{B}(x)\right] & \text { if } w=r x, r \in R, x \in M \\ 1, & \text { if } w \text { is not expressible as } w=r x .\end{cases}
$$

The following theorem gives a relation between the intuitionistic fuzzy modules on $M$ and the submodules of $M$. It is a very practical method to construct an intuitionistic fuzzy module on $M$.

Theorem 2.8. ([16]) Let $A \in I F S(M)$. Then, $A$ is an intuitionistic fuzzy module if and only if for all $\alpha, \beta \in[0,1]$ with $\alpha+\beta \leq 1$ such that $A_{(\alpha, \beta)}$ is an $R$-submodule of $M$. In particular, $A_{*}$ is an $R$-submodule of $M$. 
Definition 2.9. ([20]) Let $X$ and $Y$ be any sets and let $f: X \rightarrow Y$ be a function. An IFS $A$ of $X$ is called $f$-invariant if $f\left(x_{1}\right)=f\left(x_{2}\right) \Rightarrow \mu_{A}\left(x_{1}\right)=\mu_{A}\left(x_{2}\right)$ and $\nu_{A}\left(x_{1}\right)=\nu_{A}\left(x_{2}\right)$, where $x_{1}, x_{2} \in X$.

If $A$ is any $f$-invariant IFS of $X$, then, $f^{-1}(f(A))=A$.

Theorem 2.10. ([20]) Let $f: M \rightarrow M_{1}$ be a module homomorphism. If $A$ and $B$ are $f$-invariant IFS of $M$ and $M_{1}$, respectively. Then

1. $(f(A))_{*}=f\left(A_{*}\right)$

2. $\left(f^{-1}(B)\right)_{*}=f^{-1}\left(B_{*}\right)$.

Definition 2.11. ([18]) For $A, B \in I F S(M)$ and $C \in I F S(R)$, define the residual quotient $(A: B)$ and $(A: C)$ as follows:

$$
(A: B)=\bigcup\{D \mid D \in I F S(R) \text { such that } D \cdot B \subseteq A\}
$$

and

$$
(A: C)=\bigcup\{E \mid E \in I F S(M) \text { such that } C \cdot E \subseteq A\} .
$$

Clearly, $(A: B) \in I F S(R)$ and $(A: C) \in I F S(M)$.

Theorem 2.12. ([18]) For $A, B \in I F S(M)$ and $C \in I F S(R)$. Then, we have

(i) $(A: B)=\bigcup\left\{r_{(\alpha, \beta)}: r \in R, \alpha, \beta \in[0,1], \alpha+\beta \leq 1\right.$ such that $\left.r_{(\alpha, \beta)} \cdot B \subseteq A\right\}$

(ii) $(A: C)=\bigcup\left\{x_{(\alpha, \beta)}: x \in M, \alpha, \beta \in[0,1], \alpha+\beta \leq 1\right.$ such that $\left.C \cdot x_{(\alpha, \beta)} \subseteq A\right\}$.

Theorem 2.13. ([18]) For $A, B \in I F S(M)$ and $C \in I F S(R)$. Then, we have

(i) $(A: B) \cdot B \subseteq A$;

(ii) $C \cdot(A: C) \subseteq A$;

(iii) $C \cdot B \subseteq A \Leftrightarrow C \subseteq(A: B) \Leftrightarrow B \subseteq(A: C)$.

Theorem 2.14. ([18]) For $A_{i}(i \in J), B \in I F S(M)$ and $C \in I F S(R)$. Then, we have

(i) $\left(\bigcap_{i \in J} A_{i}: B\right)=\bigcap_{i \in J}\left(A_{i}: B\right)$;

(ii) $\left(\bigcap_{i \in J} A_{i}: C\right)=\bigcap_{i \in J}\left(A_{i}: C\right)$.

Theorem 2.15. ([18]) For $A, B \in I F S(M)$ and $C \in I F S(R)$

(i) If $A \in I F M(M)$, then, $(A: B)=\bigcup\{D \mid D \in I F I(R)$ such that $D \cdot B \subseteq A\}$;

(ii) If $C \in I F I(R)$, then, $(A: C)=\bigcup\{E \mid E \in I F S(M)$ such that $C \cdot E \subseteq A\}$.

Theorem 2.16. ([18]) If $A, B \in I F M(M)$ and $C \in I F I(R)$, then $(A: B) \in I F I(R)$ and $(A: C) \in \operatorname{IF} M(M)$. 
Let $A, B \in \operatorname{IFM}(M)$ and $C \in \operatorname{IFI}(R)$. Then, $(A: C)$ is called the residual quotient intuitionistic fuzzy submodule of $A$ and $C$ and $(A: B)$ is called the residual quotient intuitionistic fuzzy ideal of $A$ and $B$ respectively. In particular $\left(\chi_{\{\theta\}}: B\right)=\operatorname{ann}(B)$ is called the annihilator of $B$. The authors in [18] have studied the annihilator of intuitionistic fuzzy subsets of rings and modules.

Theorem 2.17. ([18]) For $A, B_{i} \in \operatorname{IF} S(M)$ and $C_{i} \in \operatorname{IF} S(R),(i \in J)$. Then, we have

(i) $\left(A: \bigcup_{i \in J} B_{i}\right)=\bigcap_{i \in J}\left(A: B_{i}\right)$;

(ii) $\left(A: \bigcup_{i \in J} C_{i}\right)=\bigcap_{i \in J}\left(A: C_{i}\right)$.

Definition 2.18. ([6]) For a non-constant $C \in I F I(R), C$ is called intuitionistic fuzzy prime ideal of $R$ if for any $x_{(p, q)}, y_{(s, t)} \in I F P(R)$, whenever $x_{(p, q)} y_{(s, t)} \subseteq C$ implies that either $x_{(p, q)} \subseteq C$ or $y_{(s, t)} \subseteq C$.

The set of intuitionistic fuzzy prime ideals of $R$ is denoted by $I F-\operatorname{Spec}(R)$.

Definition 2.19. ([6]) A non-constant IFI $A$ of a ring $R$ is called intuitionistic fuzzy maximal ideal if for any IFI $B$ of $R$, if $A \subseteq B$, then, either $B_{*}=A_{*}$, or $B=\chi_{R}$ i.e., either $B_{*}=A_{*}$, or $B_{*}=R$.

Definition 2.20. ([18]) A non-constant IFSM $A$ of an $R$-module $M$ is said to be an intuitionistic fuzzy maximal submodule if for any IFSM $B$ of $M$, if $A \subseteq B$, then, either $B_{*}=A_{*}$, or $B=\chi_{M}$, i.e., either $B_{*}=A_{*}$, or $B_{*}=M$.

Proposition 2.21. ([18]) A non-constant IFSM A of an R-module $M$ is an intuitionistic fuzzy maximal submodule if and only if $A$ is of the form

$$
\mu_{A}(x)=\left\{\begin{array}{ll}
1, & \text { if } x \in M_{0} \\
s, & \text { if } x \in M \backslash M_{0}
\end{array} ; \quad \nu_{A}(x)= \begin{cases}0, & \text { if } x \in M_{0} \\
t, & \text { if } x \in M \backslash M_{0},\end{cases}\right.
$$

where $M_{0}$ is a maximal submodule of $M$ and $s, t \in(0,1)$ such that $s+t<1$.

\section{Intuitionistic fuzzy prime submodule}

In this section, we will give a characterization of intuitionistic fuzzy prime submodules in terms of residual quotients.

Definition 3.1. For $A, B \in I F M(M), A$ is called intuitionistic fuzzy submodule of $B$ if and only if $A \subseteq B$. In particular, if $B=\chi_{M}$, then, we say that $A$ is an intuitionistic fuzzy submodule of $M$.

Definition 3.2. A non-constant intuitionistic fuzzy submodule $A$ of $B$ is said to be prime if for $C \in I F I(R)$ and $D \in I F M(M)$ such that $C \cdot D \subseteq A$, then, either $D \subseteq A$, or $C \subseteq(A: B)$. 
Definition 3.3. A non-constant intuitionistic fuzzy submodule $A$ of $M$ is said to be prime if for $C \in I F I(R)$ and $D \in I F M(M)$ such that $C \cdot D \subseteq A$, then, either $D \subseteq A$, or $C \subseteq\left(A: \chi_{M}\right)$.

The set of intuitionistic fuzzy prime submodules of $M$ is denoted by $I F-\operatorname{Spec}(M)$.

Theorem 3.4. Let $A$ be an intuitionistic fuzzy prime submodule of $M$. Then, $A^{*}$ is a prime submodule of $M$.

Proof. Here $A$ is an intuitionistic fuzzy prime submodule of $M$. Let us show $A^{*}$ is a prime submodule of $M$.

Let $r m \in A^{*}$ for some $r \in R, m \in M$. Suppose that $m \notin A^{*}$. We assume that $\mu_{A}(r m)=s$ and $\nu_{A}(r m)=t$, where $s, t \in(0,1]$ such that $s+t \leq 1$.

$$
(r m)_{(s, t)}(x)= \begin{cases}(s, t), & \text { if } x=r m \\ (0,1), & \text { if } x \neq r m\end{cases}
$$

So, $(r m)_{(s, t)} \subseteq A$. Now,

$$
\begin{aligned}
\mu_{r_{(s, t)} m_{(s, t)}}(x) & =\vee\left\{\mu_{r_{(s, t)}}(y) \wedge \mu_{m_{(s, t)}}(z): x=y z\right\} \\
& =s \\
& =\mu_{(r m)_{(s, t)}}(x) \\
& \leq \mu_{A}(x) .
\end{aligned}
$$

Thus, $\mu_{r_{(s, t)} m_{(s, t)}}(x) \leq \mu_{A}(x)$. Similarly, we get $\nu_{r_{(s, t)} m_{(s, t)}}(x) \geq \nu_{A}(x), \forall x \in M$. Thus, $r_{(s, t)} m_{(s, t)} \subseteq A$. As $A$ is an intuitionistic fuzzy prime submodule of $M$, therefore, either $m_{(s, t)} \subseteq$ $A$ or $r_{(s, t)} \subseteq\left(A: \chi_{M}\right)$. If $m_{(s, t)} \subseteq A$ Then, $\mu_{m_{(s, t)}}(x) \leq \mu_{A}(x)$ and $\nu_{m_{(s, t)}}(x) \geq \nu_{A}(x), \forall x \in M$, i.e., $s \leq \mu_{A}(x)$ and $t \geq \nu_{A}(x)$, for $x=m$. Since $x \notin A^{*}$, so $\mu_{A}(m)=0$ and $\nu_{A}(m)=1$, hence, a contradiction. This implies that $m_{(s, t)} \nsubseteq A$. Thus, $r_{(s, t)} \subseteq\left(A: \chi_{M}\right) \Rightarrow r_{(s, t)} \chi_{M} \subseteq A$.

Now, $\mu_{r_{(s, t) \chi_{M}}}(x)=\vee\left\{\mu_{r_{(s, t)}}(y) \wedge \mu_{\chi_{M}}(z) \mid x=y z\right\}=s \leq \mu_{A}(x)$. Similarly, $\nu_{r_{(s, t)} \chi_{M}}(x)=$ $t \geq \nu_{A}(x)$. Thus, $r_{(s, t)} \chi_{M} \subseteq A \Rightarrow \mu_{r_{(s, t)} \chi_{M}}(\mathrm{rm}) \leq \mu_{A}(\mathrm{rm})$ and $\nu_{r_{(s, t)} \chi_{M}}(\mathrm{rm}) \geq \nu_{A}(\mathrm{rm})$. This implies that $\mu_{A}(r m) \neq 0$ and $\nu_{A}(r m) \neq 1, \forall m \in M$. Therefore, $r m \in A^{*}, \forall m \in M$. Thus, $r M \subseteq A^{*}$, i.e., $r \in\left(A^{*}: M\right)$. Hence, $A^{*}$ is a prime submodule of $M$.

Remark 3.5. The converse of the Theorem (3.4) needs not be true, i.e., the support of an intuitionistic fuzzy submodule may be a prime submodule, but it needs not be an intuitionistic fuzzy prime submodule.

Example 3.6. Consider an IFS $A=\left(\mu_{A}, \nu_{A}\right)$ of a $Z$-module $Z$ defined by

$$
\mu_{A}(x)= \begin{cases}1 & \text { if } x=0 \\
0.5, & \text { if } x \in 2 Z-\{0\} ; \quad \nu_{A}(x)=\left\{\begin{array}{ll}
0 & \text { if } x=0 \\
0.4, & \text { if } x \in 2 Z-\{0\} \\
0, & \text { otherwise }
\end{array}\right. \text { otherwise }\end{cases}
$$

It is easy to verify that $A$ is an intuitionistic fuzzy submodule of $Z$ with $A^{*}=2 Z$, which is a prime submodule of $Z$. But we show that $A$ is not an intuitionistic fuzzy prime submodule of $Z$. 
Consider $x=4, r=5, p=0.4, q=s=0.3, t=0.1$. Then, $r_{(s, t)} x_{(p, q)}=5_{(0.3,0.1)} 4_{(0.4,0.3)}=$ $(20)_{(0.3,0,3)} \subseteq A$. But, $x_{(p, q)}=4_{(0.4,0.3)} \nsubseteq A$, for $\nu_{4_{(0.4,0.3)}}(4)=0.3 \nsupseteq \nu_{A}(4)=0.4$. Also, $r_{(s, t)}=5_{(0.3,0.1)} \nsubseteq\left(A: \chi_{M}\right)$, i.e., $5_{(0.3,0.1)} \chi_{M} \nsubseteq A$, for $\mu_{5_{(0.3,0.1)} \chi_{M}}(5)=0.3 \not \mu_{A}(5)=0$.

Theorem 3.7. If $M=R$, then, $B \in I F M(M)$, is an intuitionistic fuzzy prime submodule of $M$ if and only if $B$ is an intuitionistic fuzzy prime ideal.

Proof. Let $A$ be an intuitionistic fuzzy prime submodule of $M$. Since $A \in I F M(M)$ and $R$ is a commutative ring, $A \in I F I(R)$.

For $a_{(p, q)}, b_{(s, t)} \in \operatorname{IFP}(R), a_{(p, q)} b_{(s, t)} \subseteq A$ implies $a_{(p, q)} \subseteq A$ or $b_{(s, t)} \chi_{M} \subseteq A$.

If $a_{(p, q)} \subseteq A$, then, $A$ is an intuitionistic fuzzy prime ideal.

If $b_{(s, t)} \chi_{M} \subseteq A$, then, $\mu_{b_{(s, t)}} \chi_{M}(b m) \leq \mu_{A}(b m)$ and $\nu_{b_{(s, t)}} \chi_{M}(b m) \geq \nu_{A}(b m), \forall m \in M$. Since $R$ has identity so $b=b 1$ and $\mu_{b_{(s, t)}} \chi_{M}(b 1)=s \leq \mu_{A}(b)$ and $\nu_{b_{(s, t)}} \chi_{M}(b 1)=t \geq \nu_{A}(b)$ implies that $s=\mu_{b_{(s, t)}}(b) \leq \mu_{A}(b)$ and $t=\nu_{b_{(s, t)}}(b) \geq \nu_{A}(b)$, hence $b_{(s, t)} \subseteq A$.

Conversely, let $A$ be an intuitionistic fuzzy prime ideal of $R$. Then, $A \subset \chi_{R}$ and $A \in$ $\operatorname{IF} M(M)$. Now, let $r_{(s, t)} x_{(p, q)} \subseteq A$ for any $r_{(s, t)} \in \operatorname{IFP}(R), x_{(p, q)} \in \operatorname{IFP}(M)$.

If $x_{(p, q)} \subseteq A$, then, $A$ is an intuitionistic fuzzy prime submodule of $M$.

If $x_{(p, q)} \nsubseteq A$, then, $r_{(s, t)} \subseteq A$. Now, $\mu_{r_{(s, t)} \chi_{M}}(r m)=s \leq \mu_{A}(r) \leq \mu_{A}(r m)$ and $\nu_{r_{(s, t)} \chi_{M}}(\mathrm{rm})=t \geq \nu_{A}(r) \geq \nu_{A}(\mathrm{rm})$ by the definition of intuitionistic fuzzy ideal of $R$. Thus, $r_{(s, t)} \chi_{M} \subseteq A$, i.e., $r_{(s, t)} \in\left(A: \chi_{M}\right)$.

The following theorem, which relates intuitionistic fuzzy submodule to prime submodules of the module, will be needed in the proof of Theorem (3.10).

Theorem 3.8. Let $A$ be an intuitionistic fuzzy prime submodule of $B$. If $A_{(\alpha, \beta)} \neq B_{(\alpha, \beta)}, \alpha, \beta \in$ $[0,1]$ such that $\alpha+\beta \leq 1$, then, $A_{(\alpha, \beta)}$ is a prime submodule of $B_{(\alpha, \beta)}$.

Proof. Let $A_{(\alpha, \beta)} \neq B_{(\alpha, \beta)}$ and $r x \in A_{(\alpha, \beta)}$ for some $r \in R, x \in M$. If $r x \in A_{(\alpha, \beta)}$, then, $\mu_{A}(r x) \geq \alpha$ and $\nu_{A}(r x) \leq \beta \Rightarrow(r x)_{(\alpha, \beta)}=r_{(\alpha, \beta)} x_{(\alpha, \beta)} \subseteq A$, since $A$ is an intuitionistic fuzzy prime submodule of $B$, either $x_{(\alpha, \beta)} \subseteq A$ or $r_{(\alpha, \beta)} B \subseteq A$.

Case (i) If $x_{(\alpha, \beta)} \subseteq A$ Then, $\mu_{A}(x) \geq \alpha$ and $\nu_{A}(x) \leq \beta \Rightarrow x \in A_{(\alpha, \beta)}$.

Case (ii) Let $r_{(\alpha, \beta)} B \subseteq A$, then, for any $w \in r B_{(\alpha, \beta)}, w=r z$, for some $z \in B_{(\alpha, \beta)}$. So, $\mu_{B}(z) \geq \alpha$ and $\nu_{B}(z) \leq \beta$.

Now, $\alpha=\alpha \wedge \mu_{B}(z) \leq \sup \left\{\alpha \wedge \mu_{B}(x): w=r x\right\}=\mu_{r_{(\alpha, \beta)} B}(w) \leq \mu_{A}(w)$. Similarly, we have $\beta=\beta \vee \nu_{B}(z) \geq \inf \left\{\beta \vee \nu_{B}(x): w=r x\right\}=\nu_{r_{(\alpha, \beta)} B}(w) \geq \nu_{A}(w)$.

Thus, $w \in A_{(\alpha, \beta)}$. Thereby $r B_{(\alpha, \beta)} \subseteq A_{(\alpha, \beta)}$ i.e., $r \in\left(A_{(\alpha, \beta)}: B_{(\alpha, \beta)}\right)$. Hence $A_{(\alpha, \beta)}$ is a prime submodule of $B_{(\alpha, \beta)}$.

Corollary 3.9. Let $A$ be an intuitionistic fuzzy prime submodule of $M$. Then

$$
A_{*}=\left\{x \in M: \mu_{A}(x)=\mu_{A}(\theta) \text { and } \nu_{A}(x)=\nu_{A}(\theta)\right\}
$$

is a prime submodule of $M$.

Proof. Clear from Theorem (3.8) as $A_{(\alpha, \beta)}=A_{*}$, when $\alpha=\mu_{A}(\theta)$ and $\beta=\nu_{A}(\theta)$ and $B=$ $\chi_{M}$. 
The following theorem is the main result of this section.

Theorem 3.10. (a) Let $N$ be a prime submodule of $M$ and $\alpha, \beta \in(0,1)$ such that $\alpha+\beta<1$. If $A$ is an IFS of $M$ defined by

$$
\mu_{A}(x)=\left\{\begin{array}{ll}
1, & \text { if } x \in N \\
\alpha, & \text { if otherwise }
\end{array} ; \quad \nu_{A}(x)= \begin{cases}0, & \text { if } y \in N \\
\beta, & \text { otherwise } .\end{cases}\right.
$$

for all $x \in M$. Then, $A$ is an intuitionistic fuzzy prime submodule of $M$.

(b) Conversely, any intuitionistic fuzzy prime submodule can be obtained as in (a).

Proof. (a) Since $N$ is a prime submodule of $M, N \neq M$, we have that $A$ is a non-constant intuitionistic fuzzy submodule of $M$. We show that $A$ is an intuitionistic fuzzy prime submodule of $M$.

Suppose $r_{(s, t)} \in \operatorname{IFP}(R), x_{(p, q)} \in \operatorname{IFP}(M)$ are such that $r_{(s, t)} x_{(p, q)} \subseteq A$ and $x_{(p, q)} \nsubseteq A$. If $x_{(p, q)} \nsubseteq A$ Then, $\mu_{A}(x)=\alpha$ and $\nu_{A}(x)=\beta$, hence $x \notin N$.

If $r_{(s, t)} x_{(p, q)} \subseteq A$, then, $\mu_{(r x)_{(s \wedge p, t \vee q)}}(r x) \leq \mu_{A}(r x)$ and $\nu_{(r x)_{(s \wedge p, t \vee q)}}(r x) \geq \nu_{A}(r x) \Rightarrow s \wedge p \leq$ $\mu_{A}(r x)$ and $t \vee q \geq \nu_{A}(r x)$.

If $\mu_{A}(r x)=1$ and $\nu_{A}(r x)=0$, then, $r x \in N$. Since $x \notin N$ and $N$ is a prime submodule of $M$, we have $r M \subseteq N$. Hence $\mu_{A}(r m)=1$ and $\nu_{A}(r m)=0$, for all $m \in M$. Thus, $\mu_{r_{(s, t)} \chi_{M}}(r m)=s \leq \mu_{A}(r m)$ and $\nu_{r_{(s, t)} \chi_{M}}(r m)=t \geq \nu_{A}(r m)$.

If $\mu_{A}(r x)=\alpha$ and $\nu_{A}(r x)=\beta$, then, $s \wedge p \leq \alpha$ and $t \vee q \geq \beta$. If $p \not \leq \alpha$, then, $s \leq \alpha$ and $t \geq \beta$.

Thus, $\mu_{r_{(s, t)} \chi_{M}}(w)=s \leq \alpha \leq \mu_{A}(w)$ and $\nu_{r_{(s, t)} \chi_{M}}(w)=t \geq \beta \geq \nu_{A}(w)$, for all $w \in M$. Therefore, $r_{(s, t)} \chi_{M} \subseteq A$, i.e., $r_{(s, t)} \in\left(A: \chi_{M}\right)$. Hence $A$ is an intuitionistic fuzzy prime submodule of $M$.

(b) Let $A$ be an intuitionistic fuzzy prime submodule of $M$. We show that $A$ is of the form

$$
\mu_{A}(x)=\left\{\begin{array}{ll}
1, & \text { if } x \in N \\
\alpha, & \text { if otherwise }
\end{array} ; \quad \nu_{A}(x)= \begin{cases}0, & \text { if } y \in N \\
\beta, & \text { otherwise }\end{cases}\right.
$$

for all $x \in M$, where $\alpha, \beta \in(0,1)$ such that $\alpha+\beta<1$.

Since $A$ is a non-constant intuitionistic fuzzy prime submodule of $M$, so $A_{*}$ is a prime submodule of $M$.

Claim (1) $A$ has two values.

Since $A_{*}$ is a prime submodule of $M, A_{*} \neq M$, then, there exists $z \in M \backslash A_{*}$. We will show that $\mu_{A}(y)=\mu_{A}(z)<\mu_{A}(\theta)$ and $\nu_{A}(y)=\nu_{A}(z)>\nu_{A}(\theta)$, for all $y \in M$ such that $y \in A_{*}$. Then, $z \in A_{*} \Rightarrow \mu_{A}(z)<1=\mu_{A}(\theta)$ and $\nu_{A}(z)>0=\nu_{A}(\theta)$ so $z_{(1,0)} \notin A$ and $z_{\left(\mu_{A}(z), \nu_{A}(z)\right)}=$ $z_{(1,0)} 1_{\left(\mu_{A}(z), \nu_{A}(z)\right)} \in A$. Thus, $1_{\left(\mu_{A}(z), \nu_{A}(z)\right)} \chi_{M} \subseteq A$, since $w=1 . w$, for all $w \in M$, we have $\mu_{A}(z) \leq \mu_{A}(w)$ and $\nu_{A}(z) \geq \nu_{A}(w)$.

Let $w=y$. Then, $\mu_{A}(z) \leq \mu_{A}(y)$ and $\nu_{A}(z) \geq \nu_{A}(y)$. Similarly, $\mu_{A}(y) \leq \mu_{A}(z)$ and $\nu_{A}(y) \geq \nu_{A}(z)$. Hence $\mu_{A}(z)=\mu_{A}(y)$ and $\nu_{A}(z)=\nu_{A}(y)$. 
Claim (2) Let $\mu_{A}(z)=\alpha$ and $\nu_{A}(z)=\beta$, where $\alpha, \beta \in(0,1)$ such that $\alpha+\beta<1$. First, let $p, q, s, t \in(0,1]$ such that $p+q \leq 1, s+t \leq 1$. Consider $s \wedge p \leq \alpha$ and $t \vee q \geq \beta$ and let $p \$ \alpha$ Then, $s \leq \alpha$ and $t \geq \beta$.

Suppose $x \in M \backslash A_{*}$, then, $x_{(p, q)} \nsubseteq A$. Hence $1_{(s, t)} x_{(p, q)}=x_{(s \wedge p, t \vee q)} \subseteq A \Rightarrow 1_{(s, t)} \chi_{M} \subseteq A$, and for all $w \in M, \mu_{1_{(s, t)} \chi_{M}}(w) \leq \mu_{A}(w)$ and $\nu_{1_{(s, t)} \chi_{M}}(w) \geq \nu_{A}(w)$. Let $w=x$. Then, $s=\mu_{1_{(s, t)} \chi_{M}}(w) \leq \mu_{A}(x)=\alpha$ and $t=\nu_{1_{(s, t)} \chi_{M}}(w) \geq \nu_{A}(x)=\beta$. Thus, every intuitionistic fuzzy prime submodule of $M$ is of the form

$$
\mu_{A}(x)=\left\{\begin{array}{ll}
1, & \text { if } x \in N \\
\alpha, & \text { if otherwise }
\end{array} ; \quad \nu_{A}(x)= \begin{cases}0, & \text { if } x \in N \\
\beta, & \text { otherwise }\end{cases}\right.
$$

for all $x \in M$, where $\alpha, \beta \in(0,1)$ such that $\alpha+\beta<1$ and $N$ is a prime submodule of $M$.

This theorem is particularly useful in deciding whether or not an intuitionistic fuzzy submodule is prime. The following example illustrates this.

Example 3.11. Let $M=Z$ be a module over $R=Z$. Then

$$
\mu_{A}(x)=\left\{\begin{array}{ll}
1, & \text { if } x \in 3 Z \\
0.2, & \text { if otherwise }
\end{array} ; \quad \nu_{A}(x)= \begin{cases}0, & \text { if } x \in 3 Z \\
0.7, & \text { otherwise }\end{cases}\right.
$$

is an intuitionistic fuzzy prime submodule of $Z$, since $3 Z$ is a prime submodule of $Z$.

Proposition 3.12. Let $A, B$ be two intuitionistic fuzzy submodules of $M$. Then, $A \cap B$ is also an intuitionistic fuzzy prime submodule of $M$.

Proof. Let $C \in I F I(R), D \in I F M(M)$ such that $C \cdot D \subseteq A \cap B$. To show that $A \cap B$ is also intuitionistic fuzzy prime submodule of $M$, we show that either $D \subseteq A \cap B$ or $C \subseteq\left(A \cap B: \chi_{M}\right)$.

Since $C \cdot D \subseteq A \cap B \Rightarrow C \cdot D \subseteq A$ and $C \cdot D \subseteq B$. As $A$ and $B$ are intuitionistic fuzzy prime submodules of $M$. Therefore, either $D \subseteq A$ or $C \subseteq\left(A: \chi_{M}\right)$ and $D \subseteq B$ or $C \subseteq\left(B: \chi_{M}\right) \Rightarrow D \subseteq A \cap B$ or $C \subseteq\left(A: \chi_{M}\right) \cap\left(B: \chi_{M}\right)=\left(A \cap B: \chi_{M}\right)$.

Corollary 3.13. If $A_{i}(i \in J)$ are intuitionistic fuzzy prime submodules of $M$, then, $\bigcap_{i \in J} A_{i}$ is also an intuitionistic fuzzy prime submodule of $M$.

Theorem 3.14. Let $B \in I F S(M)$ and $A \in I F-\operatorname{Spec}(M)$.

(i) If $B \subseteq A$, then, $(A: B)=\chi_{R}$, and

(ii) If $B \nsubseteq A$, then, $(A: B)=\left(A: \chi_{M}\right)$.

Proof. (i) Since $B \subseteq A$, then, for any $C \in I F I(R)$, we have $C \cdot B \subseteq \chi_{R} \cdot B \subseteq \chi_{R} \cdot A=A$. Therefore, $(A: B)=\bigcup\{C \mid C \in I F I(R)$ such that $C \cdot B \subseteq A\}=\chi_{R}$.

(ii) Suppose $B \nsubseteq A$. If $C \cdot B \subseteq A$ for some $C \in I F I(R)$. As $A$ is an intuitionsitic fuzzy prime submodule of $M$, we have $C \subseteq\left(A: \chi_{M}\right)$. Thus, $(A: B)=\bigcup\{C \mid C \in I F I(R)$ such that $C \cdot B \subseteq A\} \subseteq\left(A: \chi_{M}\right)$. But $\left(A: \chi_{M}\right) \subseteq(A: B)$ always. Therefore, $(A: B)=\left(A: \chi_{M}\right)$. 
Theorem 3.15. Let $A \in I F M(M)$ and $C \in I F I(R)$. If $A$ is an intuitionistic fuzzy prime submodule of $M$, then

(i) if $C \nsubseteq\left(A: \chi_{M}\right)$, then, $(A: C)=A$, and

(ii) if $C \subseteq\left(A: \chi_{M}\right)$, then, $(A: C)=\chi_{M}$.

Proof. (i) Let $C \nsubseteq\left(A: \chi_{M}\right)$ and $C \cdot B \subseteq A$ for some $B \in I F M(M)$. As $A$ is intuitionistic fuzzy prime submodule of $M$ we have $B \subseteq A$. Therefore, $(A: C)=\bigcup\{B: B \in I F I(R)$ such that $C$. $B \subseteq A\} \subseteq A$. Clearly, $C \cdot A \subseteq \chi_{R} \cdot A=A$ and so, $A \subseteq(A: C)$. Hence $(A: C)=A$.

(ii) Assume that $C \subseteq\left(A: \chi_{M}\right)$. Then, $C \cdot \chi_{M} \subseteq\left(A: \chi_{M}\right) \cdot \chi_{M} \subseteq A$. Thus, $C \cdot B \subseteq C \cdot \chi_{M} \subseteq A$ for all $B \in \operatorname{IFI}(M)$. Hence $(A: C)=\chi_{M}$.

Theorem 3.16. Let $A$ be an intuitionistic fuzzy prime submodule of $M$ and $B \in I F M(M)$. If $(A: B) \neq \chi_{R}$, then, $(A: B)$ is an intuitionistic fuzzy prime ideal of $R$.

Proof. We assume $(A: B) \neq \chi_{R}$. Then, $B \nsubseteq A$ and so by Theorem 3.14 we have $(A: B)=\left(A: \chi_{M}\right)$. Let $C, D \in I F I(R)$ be such that $C \cdot D \subseteq(A: B)$ and $C \nsubseteq(A: B)$. Now $C \cdot D \subseteq(A: B)$ gives $(C D) B \subseteq A$. This implies $C(D B) \subseteq A$. As $A$ is an intuitionistic fuzzy prime submodule of $M$, this implies either $C \subseteq\left(A: \chi_{M}\right)$ or $D B \subseteq A$. If $C \subseteq\left(A: \chi_{M}\right)$ Then, $C \chi_{M} \subseteq A$, also $B \subseteq \chi_{M}$. This implies $C B \subseteq C \chi_{M} \subseteq A$, i.e., $C B \subseteq A$ this further implies that $C \subseteq(A: B)$, which is a contradiction. So $D B \subseteq A$ and this implies $D \subseteq(A: B)$. Thus, $C \cdot D \subseteq(A: B)$ implies that either $C \subseteq(A: B)$ or $D \subseteq(A: B)$. Hence $(A: B)$ is an intuitionistic fuzzy prime ideal of $R$.

Corollary 3.17. If $A$ is an intuitionistic fuzzy prime submodule of $M$, then, $\left(A: \chi_{M}\right)$ is an intuitionistic fuzzy prime ideal of $R$.

Theorem 3.18. Let $A \in I F M(M)$ and $C \in I F I(R)$. If $A$ is an intuitionistic fuzzy prime submodule of $M$ and $(A: C) \neq \chi_{M}$, then, $(A: C)$ is an intuitionistic fuzzy prime submodule of $M$.

Proof. Let $D \in I F I(R), B \in I F I(M)$ and $D B \subseteq(A: C)$. Then, $D(C B)=C(D B)$ $\subseteq C(A: C) \subseteq A$. As $A$ is an intuitionistic fuzzy prime submodule of $M$, therefore, either $C B \subseteq A$, or $D \subseteq\left(A: \chi_{M}\right)$. Also, $C B \subseteq A$ gives $B \subseteq(A: C)$. As $(A: C) \neq \chi_{M}$, we get $C \chi_{M} \nsubseteq A$. Now,

$$
\begin{aligned}
\left((A: C): \chi_{M}\right) & =\bigcap\left\{D \mid D \in \operatorname{IFI}(R), D \chi_{M} \subseteq(A: C)\right\} \\
& =\bigcap\left\{D \mid D \in \operatorname{IFI}(R), C\left(D \chi_{M}\right) \subseteq A\right\} \\
& =\bigcap\left\{D \mid D \in \operatorname{IFI}(R), D\left(C \chi_{M}\right) \subseteq A\right\} \\
& =\left(A:\left(C: \chi_{M}\right)\right) .
\end{aligned}
$$

Thus, $\left((A: C): \chi_{M}\right)=\left(A:\left(C: \chi_{M}\right)\right)$, so by Theorem 3.15 we have $\left((A: C): \chi_{M}\right)$ $=\left(A: \chi_{M}\right)$. This gives that $B \subseteq(A: C)$ or $\left.D \subseteq\left(A: \chi_{M}\right)=\left((A: C): \chi_{M}\right)\right)$. Therefore, $(A: C)$ is an intuitionistic fuzzy submodule of $M$. 
Corollary 3.19. If $A$ is an intuitionistic fuzzy prime submodule of $M$, then, $\left(A: \chi_{R}\right)$ is an intuitionistic fuzzy prime submodule of $M$.

Proposition 3.20. If a non-constant intuitionistic fuzzy maximal submodule $A$ of an $R$-module $M$, then, $A$ is an intuitionistic fuzzy prime submodule of $M$.

Proof. By Proposition (2.21) $A$ is of the form

$$
\mu_{A}(x)=\left\{\begin{array}{ll}
1, & \text { if } x \in M_{0} \\
s, & \text { if } x \in M \backslash M_{0}
\end{array} ; \quad \nu_{A}(x)= \begin{cases}0, & \text { if } x \in M_{0} \\
t, & \text { if } x \in M \backslash M_{0},\end{cases}\right.
$$

where $M_{0}$ is a maximal submodule of $M$ and $s, t \in(0,1)$ such that $s+t<1$.

Also, by Lemma 2.1 if $M_{0}$ is a maximal submodule of $M$, then, $M_{0}$ is a prime submodule of $M$. Then, the result follows from Theorem 3.10.

Proposition 3.21. If $A \in I F M(M)$ is such that $\left(A: \chi_{M}\right)$ is an intuitionistic fuzzy maximal ideal of $R$, then, $A$ is an intuitionistic fuzzy prime submodule of $M$.

Proof. Let $B \in I F I(R)$ be such that $\left(A: \chi_{M}\right) \subseteq B$. As $\left(A: \chi_{M}\right)$ is an intuitionistic fuzzy maximal ideal of $R$. Therefore, either $B_{*}=\left(A: \chi_{M}\right)_{*}$ or $B_{*}=R$, i.e., either $B_{*}=\left(A_{*}: M\right)$ or $B_{*}=R$. This implies that $\left(A_{*}: M\right)$ is a maximal ideal of $R$. Then, by Lemma 2.1 we have $A_{*}$ is maximal prime submodule of $M$. Then, from Theorem 3.10 (b), we get that $A$ is an intuitionistic fuzzy prime submodule of $M$.

\section{Intuitionistic fuzzy prime submodules of homomorphic modules}

In this section, we investigate the behaviour of intuitionistic fuzzy prime submodules under an $R$-module epimorphism. Firstly, we recall the definition of image and inverse image of an intuitionistic fuzzy subset under an $R$-module homomorphism. From now on, $M$ and $M_{1}$ are $R$-modules.

Definition 4.1. Let $f$ be an $R$-module homomorphism from $M$ to $M_{1}, A \in I F S(M)$ and $B \in \operatorname{IFS}\left(M_{1}\right)$. Then, $\forall w \in M_{1}, f(A) \in \operatorname{IFS}\left(M_{1}\right)$ and $f^{-1}(B) \in \operatorname{IFS}(M)$ are defined by

$$
f(A)(w)= \begin{cases}\left(\sup \left\{\mu_{A}(m): m \in f^{-1}(w)\right\}, \operatorname{Inf}\left\{\nu_{A}(m): m \in f^{-1}(w)\right\}\right), & \text { if } f^{-1}(w) \neq \phi \\ (0,1), & \text { otherwise }\end{cases}
$$

Also, $f^{-1}(B)(m)=\left(\mu_{B}(f(m)), \nu_{B}(f(m))\right), \forall m \in M$.

In the next two theorems we show that both the image and the inverse image of an intuitionistic fuzzy prime submodules under an $R$-module epimorphism are again intuitionistic fuzzy prime submodules. 
Theorem 4.2. Let $f$ be an $R$-module epimorphism from $M$ to $M_{1}$. If $A$ is an intuitionistic fuzzy prime submodule of $M$ such that $A$ is $f$-invariant, then, $f(A)$ is an intuitionistic fuzzy prime submodule of $M_{1}$.

Proof. Since $A$ is an intuitionistic fuzzy prime submodule of $M$ and $f: M \rightarrow M_{1}$ is an $R$-module epimorphism. Therefore, $f(A)$ is an intuitionistic fuzzy submodule of $M_{1}$ (see [15]).

Next, we show that $f(A)$ is an intuitionistic fuzzy prime submodule of $M_{1}$. Since $A$ is an intuitionistic fuzzy prime submodule of $M$, so $A$ is of the form

$$
\mu_{A}(x)=\left\{\begin{array}{ll}
1, & \text { if } x \in N \\
\alpha, & \text { if otherwise }
\end{array} ; \quad \nu_{A}(x)= \begin{cases}0, & \text { if } x \in N \\
\beta, & \text { otherwise }\end{cases}\right.
$$

for all $x \in M$, where $\alpha, \beta \in(0,1)$ such that $\alpha+\beta<1$ and $N=A_{*}$ is a prime submodule of $M$.

We first claim that $f\left(A_{*}\right)$ is a prime submodule of $M_{1}$.

For all $r \in R, w \in M_{1}$,rw $\in f\left(A_{*}\right)$, there exists $z \in A_{*}$ such that $r w=f(z)$. Since $f$ is an epimorphism there exists $m \in M$ such that $r w=r f(m)=f(r m)=f(z)$. As $A$ is $f$-invariant, so $\mu_{A}(r m)=\mu_{A}(z)=\mu_{A}(\theta)$ and $\nu_{A}(r m)=\nu_{A}(z)=\nu_{A}(\theta)$. This implies that $r m \in A_{*}$. As $A_{*}$ is a prime submodule of $M$, so either $m \in A_{*}$ or $r M \subseteq A_{*}$.

If $m \in A_{*}$, then, $w=f(m) \in f\left(A_{*}\right)$ and if $r M \subseteq A_{*}$, then, $r M_{1}=f(r M) \subseteq f\left(A_{*}\right)$.

Thus, $f\left(A_{*}\right)$ is a prime submodule of $M_{1}$, so by Theorem 3.10 , for all $w \in M_{1}$,

$$
\mu_{f(A)}(w)=\left\{\begin{array}{ll}
1, & \text { if } w \in f\left(A_{*}\right) \\
\alpha, & \text { if otherwise }
\end{array} ; \quad \nu_{f(A)}(w)= \begin{cases}0, & \text { if } w \in f\left(A_{*}\right) \\
\beta, & \text { otherwise }\end{cases}\right.
$$

Hence $f(A)$ is an intuitionistic fuzzy prime submodule of $M_{1}$.

Example 4.3. Let $f$ be a homomorphism from $Z$ to $Z$, defined by $f(x)=2 x$, and let

$$
\mu_{A}(x)=\left\{\begin{array}{ll}
1, & \text { if } x \in 3 Z \\
0.2, & \text { if otherwise }
\end{array} ; \quad \nu_{A}(x)= \begin{cases}0, & \text { if } x \in 3 Z \\
0.7, & \text { otherwise }\end{cases}\right.
$$

be an intuitionistic fuzzy prime submodule of $Z$. Then

$f(A)(0)=\left(\sup \left\{\mu_{A}(x) \mid f(n)=0\right\}, \inf \left\{\nu_{A}(x) \mid f(n)=0\right\}\right)=\left(\mu_{A}(0), \nu_{A}(0)\right)=(1,0)$ and $f(A)(1)=\left(\sup \left\{\mu_{A}(x) \mid f(n)=1\right\}, \inf \left\{\nu_{A}(x) \mid f(n)=1\right\}\right)=(0,1)\left[\right.$ As $\left.f^{-1}(1)=\emptyset\right]$. Similarly, we can find that $f(A)(3)=f(A)(5)=(0,1)$ and $f(A)(2)=f(A)(4)=(0.2,0.7)$ and so on we get

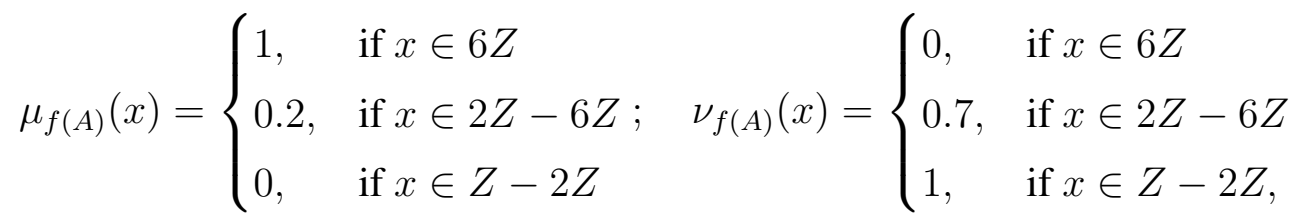


is not an intuitionistic prime fuzzy submodule of $Z$. This shows that the assumption that $f$ is an epimorphism in Theorem (4.2) cannot be dropped.

Theorem 4.4. Let $f$ be an R-module epimorphism from $M$ to $M_{1}$. If $B$ is an intuitionistic fuzzy prime submodule of $M_{1}$, then, $f^{-1}(B)$ is an intuitionistic fuzzy prime submodule of $M$.

Proof. Let $B$ be an intuitionistic fuzzy prime submodule of $M_{1}$. Then

$$
\mu_{B}(x)=\left\{\begin{array}{ll}
1, & \text { if } x \in B_{*} \\
\alpha, & \text { if otherwise }
\end{array} ; \quad \nu_{B}(x)= \begin{cases}0, & \text { if } x \in B_{*} \\
\beta, & \text { otherwise }\end{cases}\right.
$$

for all $x \in M_{1}$, where $\alpha, \beta \in(0,1)$ such that $\alpha+\beta<1$ and $B_{*}$ is a prime submodule of $M_{1}$.

We first show that $f^{-1}\left(B_{*}\right)$ is a prime submodule of $M$.

For all $r \in R, m \in M$, if $r m \in f^{-1}\left(B_{*}\right) \Rightarrow f(r m) \in B_{*}$, i.e., $r f(m) \in B_{*}$. As $B_{*}$ is a prime submodule of $M_{1}$. Therefore, either $f(m) \in B_{*}$ or $r M_{1} \subseteq B_{*}$.

If $f(m) \in B_{*}$, then, $m \in f^{-1}\left(B_{*}\right)$ and if $r M_{1} \subseteq B_{*}$, then, $r f(M)=f(r M) \subseteq B_{*} \Rightarrow r M \subseteq$ $f^{-1}\left(B_{*}\right)$. Hence for all $x \in M$, we have

$$
\mu_{f^{-1}(B)}(x)=\left\{\begin{array}{ll}
1, & \text { if } x \in f^{-1}\left(B_{*}\right) \\
\alpha, & \text { if otherwise }
\end{array} ; \quad \nu_{f^{-1}(B)}(x)= \begin{cases}0, & \text { if } x \in f^{-1}\left(B_{*}\right) \\
\beta, & \text { otherwise }\end{cases}\right.
$$

Hence $f^{-1}(B)$ is an intuitionistic fuzzy prime submodule of $M$.

Theorem 4.5. Let $M$ and $M_{1}$ be two modules over $R$ and let $f$ be an epimorphism of $M$ onto $M_{1}$ (i) If $A$ is an intuitionistic fuzzy prime submodule of $M$ and is also f-invariant, then

$$
\left(A: \chi_{M}\right)=\left(f(A): \chi_{M_{1}}\right)
$$

(ii) If $B$ is an intuitionistic fuzzy prime submodule of $M_{1}$, then

$$
\left(B: \chi_{M_{1}}\right)=\left(f^{-1}(B): \chi_{M}\right) .
$$

Proof. (i) We assume that $A$ is an intuitionistic fuzzy prime submodule of $M$ and is also an $f$-invariant. Then, $f(A)$ is an intuitionistic fuzzy prime submodule of $M_{1}$.

Let $r \in\left(f\left(A_{*}\right): M_{1}\right)$. Then, $r M_{1} \subseteq f\left(A_{*}\right) \Rightarrow r f(M) \subseteq f\left(A_{*}\right) \Rightarrow f(r M) \subseteq f\left(A_{*}\right)$. Let $x=r m \in r M, m \in M$. Then, $f(r m) \in f(r M)$. Then, $f(x)=f(r m) \subseteq f\left(A_{*}\right)$. This implies that $f(x)=f(z)$, for some $z \in A_{*}$. As $A$ is $f$-invariant so $\mu_{A}(x)=\mu_{A}(z)=\mu_{A}(\theta)$ and $\nu_{A}(x)=\nu_{A}(z)=\nu_{A}(\theta) \Rightarrow x \in A_{*}$. From this we get that $r M \subseteq A_{*}$ and this implies that $r \in\left(A_{*}: M\right)$, so $\left(f\left(A_{*}\right): M_{1}\right) \subseteq\left(A_{*}: M\right)$.

Again let $p \in\left(A_{*}: M\right)$. Then, $p M \subseteq A_{*}$. From this we get $f(p M) \subseteq f\left(A_{*}\right)$. So, $p f(M)$ $\subseteq f\left(A_{*}\right)$, i.e., $p M_{1} \subseteq f\left(A_{*}\right)$, as $f$ is an epimorphism. This implies that $p \in\left(f\left(A_{*}\right): M_{1}\right)$. Thus, $\left(A_{*}: M\right)=\left(f\left(A_{*}\right): M_{1}\right)$. Hence $\left(A: \chi_{M}\right)=\left(f(A): \chi_{M_{1}}\right)$.

(ii) We assume that $B$ is an intuitionistic fuzzy prime submodule of $M_{1}$. Then, $f^{-1}(B)$ is an intuitionistic fuzzy prime submodule of $M$. 
Let $r \in\left(f^{-1}\left(B_{*}\right): M\right)$. Then, $r M \subseteq f^{-1}\left(B_{*}\right) \Rightarrow f(r M) \subseteq B_{*}$, i.e., $r f(M) \subseteq B_{*}$ as $f$ is an epimorphism, which implies that $r M_{1} \subseteq B_{*}$. This implies that $r \in\left(B_{*}: M_{1}\right)$. So, $\left(f^{-1}(B): M\right) \subseteq\left(B_{*}: M_{1}\right)$.

Again, let $p \in\left(B_{*}: M_{1}\right)$. Then, $p M_{1} \subseteq B_{*}$. This implies that $p f(M) \subseteq B_{*}$, as $f$ is an epimorphism, so $f(p M) \subseteq B_{*}$, i.e., $p M \subseteq f^{-1}\left(B_{*}\right) \Rightarrow p \in\left(f^{-1}\left(B_{*}\right): M\right)$.

Thus, $\left(B_{*}: M_{1}\right) \subseteq\left(f^{-1}\left(B_{*}\right): M\right)$. Thus, $\left(f^{-1}\left(B_{*}\right): M\right)=\left(B_{*}: M_{1}\right)$. Therefore, $\left(B: \chi_{M_{1}}\right)=\left(f^{-1}(B): \chi_{M}\right)$.

\section{Conclusions}

In this paper we have studied intuitionistic fuzzy prime submodules with the help of residual quotient of intuitionistic fuzzy subset of rings and modules. We also investigate some important characterizations of intuitionistic fuzzy prime submodule. A relationship between intuitionistic fuzzy maximal submodules and intuitionistic fuzzy prime submodules has been developed. The homomorphic behaviour of intuitionistic fuzzy prime submodules has been investigated. For further study, we shall investigate the decomposition of intuitionistic fuzzy prime submodules and try to explore the Goldie like structure in intuitionistic fuzzy setting.

\section{Acknowledgements}

The second author would like to thank IKG PT University, Jalandhar for providing the opportunity to do research work.

\section{References}

[1] Ameri, R., \& Mahjoob, R. (2008). Spectrum of prime L-submodules. Fuzzy Sets and Systems, 159, 1107-1115.

[2] Amini, A., Amini, B., \& Sharif, H. (2006). Prime and primary submodule of certain modules. Czechoslovak Mathematical Journal, 56 (131), 641-648.

[3] Atanassov K. T. (1983). Intuitionistic Fuzzy Sets, VII ITKR Session, Sofia, 20-23 June 1983 (Deposed in Centr. Sci.-Techn. Library of the Bulg. Acad. of Sci., 1697/84) (in Bulgarian). Reprinted: Int. J. Bioautomation, 2016, 20(S1), S1-S6.

[4] Atanassov K. T. (1986). Intuitionistic fuzzy sets. Fuzzy Sets and Systems, 20 (1), 87-96.

[5] Atanassov K. T. (1999). Intuitionistic Fuzzy Sets: Theory and Applications, Studies on Fuzziness and Soft Computing, Vol. 35, Physica-Verlag, Heidelberg.

[6] Bakhadach, I., Melliani, S., Oukessou, M., \& Chadli, L. S. (2016). Intuitionistic fuzzy ideal and intuitionistic fuzzy prime ideal in a ring, Notes on Intuitionistic Fuzzy Sets, 22 (2), $59-63$. 
[7] Basnet, D. K. (2011). Topics in Intuitionistic Fuzzy Algebra, Lambert Academic Publishing.

[8] Biswas, R. (1989). Intuitionistic fuzzy subgroup, Mathematical Forum, X, 37-46.

[9] Bland Paul, E. (2012). Rings and Their Modules. Deutsche Nationalbibliothek, Germany.

[10] Davvaz, B., Dudek, W. A., \& Jun, Y. B. (2006). Intuitionistic fuzzy Hv-submodules. Information Science, 176, 285-300.

[11] Hur, K., Jang, S. Y., \& Kang, H. W. (2005). Intuitionistic Fuzzy Ideals of a Ring. Journal of the Korea Society of Mathematical Education, Series B, 12 (3), 193-209.

[12] Isaac, P., John, P. P. (2011). On Intuitionistic Fuzzy Submodules of a Module. Int. J. of Mathematical Sciences and Applications, 1 (3), 1447-1454.

[13] John, P. P., \& Isaac, P. (2012). IFSM's of an R-Module - A Study. International Mathematical Forum, 19 (7), 935-943.

[14] Mordeson, J. N., \& Malik, D. S. (1998). Fuzzy Commutative Algebra, World Scientific Publishing Co-Pvt. Ltd.

[15] Rahman, S., \& Saikia, H. K. (2012). Some aspects of Atanassov's intuitionistic fuzzy submodules, Int. J. Pure and Appl. Mathematics, 77 (3), 369-383.

[16] Sharma, P. K. (2013). ( $\alpha, \beta)$-Cut of intuitionistic fuzzy modules-II, Int. J. of Mathematical Sciences and Applications, 3 (1), 11-17.

[17] Sharma, P. K., \& Kaur, T. (2015). Intuitionistic fuzzy G-modules. Notes on Intuitionistic Fuzzy Sets, 21 (1), 6-23.

[18] Sharma, P. K., \& Kaur, G. (2017). Residual quotient and annihilator of intuitionistic fuzzy sets of ring and module, . International Journal of Computer Sciences and Information Techonology, 9(4), 1-15.

[19] Sharma, P. K., \& Kaur, G. (2017). Intuitionistic fuzzy prime spectrum of a ring. CiiT International Journal of Fuzzy Systems, 9 (8), 167-175.

[20] Sharma, P. K., \& Kaur, G. (2018). On the intuitionistic fuzzy polynomial ideals of a ring. Notes on Intuitionistic Fuzzy Sets, 24 (1), 48-59.

[21] Zadeh, L. A. (1965). Fuzzy Sets. Information and Control, 8, 338-353. 